\title{
The Cauchy Problem for Space-Time Monopole Equations in Temporal and Spatial Gauge
}

\author{
Hyungjin Huh and Jihyun Yim \\ Department of Mathematics, Chung-Ang University, Seoul 156-756, Republic of Korea \\ Correspondence should be addressed to Hyungjin Huh; huh@cau.ac.kr
}

Received 7 October 2016; Revised 6 January 2017; Accepted 22 January 2017; Published 20 February 2017

Academic Editor: Ming Mei

Copyright (c) 2017 Hyungjin Huh and Jihyun Yim. This is an open access article distributed under the Creative Commons Attribution License, which permits unrestricted use, distribution, and reproduction in any medium, provided the original work is properly cited.

We prove global existence of solution to space-time monopole equations in one space dimension under the spatial gauge condition $A_{1}=0$ and the temporal gauge condition $A_{0}=0$.

\section{Introduction}

In the current article, we study the following space-time monopole equations in one space dimension:

$$
\begin{aligned}
\partial_{t} \phi+\left[A_{0}, \phi\right]+\partial_{x} N+\left[A_{1}, N\right] & =0, \\
\partial_{t} N+\left[A_{0}, N\right]+\partial_{x} \phi+\left[A_{1}, \phi\right] & =0, \\
\partial_{t} A_{1}-\partial_{x} A_{0}+\left[A_{0}, A_{1}\right] & =[N, \phi] .
\end{aligned}
$$

Here $\phi, N, A=\left(A_{0}, A_{1}\right): \mathbb{R}^{1+1} \rightarrow \mathbf{g}$, where $\mathbf{g}$ is a Lie algebra of a matrix Lie group such as $\mathrm{SO}(n), \mathrm{SU}(n)$ with Lie bracket $[\cdot, \cdot]$. We denote space-time derivatives by $\partial_{0}=\partial_{t}, \partial_{1}=\partial_{x}$.

The space-time monopole equations in $\mathbb{R}^{2+1}$ can be written as follows:

$$
\begin{aligned}
& \partial_{0} \phi+\partial_{1} A_{2}-\partial_{2} A_{1}=\left[\phi, A_{0}\right]+\left[A_{2}, A_{1}\right], \\
& \partial_{0} A_{2}+\partial_{1} \phi-\partial_{2} A_{0}=\left[A_{2}, A_{0}\right]+\left[\phi, A_{1}\right], \\
& \partial_{0} A_{1}-\partial_{1} A_{0}-\partial_{2} \phi=\left[A_{2}, \phi\right]+\left[A_{1}, A_{0}\right] .
\end{aligned}
$$

Equation (1) is obtained by the dimensional reduction of system (2). More precisely, we consider (2) independent of the $y$ coordinate and renaming $A_{2}$ as $N$ to get (1). The spacetime monopole system (2) is a nonabelian gauge field theory and can be derived by dimensional reduction from anti-selfdual Yang-Mills equations; see [1], for instance. The system is an example of a completely integrable system and has an equivalent formulation as a Lax pair. It was first introduced by Ward in [2] as a hyperbolic analog of Bogomol'nyi equations and discussed from the point of view of twistors.

System (1) is invariant under the rescaling

$$
\begin{aligned}
\phi^{\lambda}(t, x) & =\lambda \phi(\lambda t, \lambda x), \\
A_{\mu}^{\lambda}(t, x) & =\lambda A_{\mu}(\lambda t, \lambda x), \\
N^{\lambda}(t, x) & =\lambda N(\lambda t, \lambda x),
\end{aligned}
$$

from which we deduce a scale invariant Lebesgue space $L^{1}(\mathbb{R})$ and Sobolev space $\dot{H}^{-1 / 2}(\mathbb{R})$. Another important property of system (1) is an invariance under the gauge transformation

$$
\begin{aligned}
& \phi \longrightarrow g \phi g^{-1}, \\
& A \longrightarrow g A g^{-1}+g d g^{-1}, \\
& N \longrightarrow g N g^{-1},
\end{aligned}
$$

where $g: \mathbb{R}^{1+1} \rightarrow \mathbf{G}$ is smooth and compactly supported map into Lie group $\mathbf{G}$.

A broad survey on the space-time monopole equations is given in [1]. In particular, using the inverse scattering transform, they have shown global existence and uniqueness up to a gauge transformation for small initial data in $W^{2,1}\left(\mathbb{R}^{2}\right)$. The survey [1] also contained a number of other interesting 
results related to the space-time monopole equations. Czubak showed in [3] that the space-time monopole system in Coulomb gauge is locally well-posed for small initial data in $H^{s}\left(\mathbb{R}^{2}\right)$ with $s>1 / 4$. The Cauchy problem of the spacetime monopole equations in $\mathbb{R}^{2+1}$ under the Lorenz gauge condition was discussed in $[4,5]$. In particular almost critical local well-posedness has been proved in [5]. The existence of global solution in $\mathbb{R}^{1+1}$, under Lorenz gauge condition $\partial_{0} A_{0}-\partial_{1} A_{1}=0$, was proved in [6] for Lebesgue space $L^{p}(\mathbb{R})$ with $1 \leq p \leq \infty$. In the current article, we will consider two gauge conditions $A_{1}=0$ and $A_{0}=0$.

First we impose the spatial gauge condition $A_{1}=0$. Then system (1) becomes

$$
\begin{aligned}
\partial_{t} \phi+\partial_{x} N & =\left[\phi, A_{0}\right], \\
\partial_{t} N+\partial_{x} \phi & =\left[N, A_{0}\right], \\
-\partial_{x} A_{0} & =[N, \phi] .
\end{aligned}
$$

With the notations of $\phi+N=u$ and $\phi-N=v$, system (5) can be rewritten as

$$
\begin{aligned}
\partial_{t} u+\partial_{x} u & =\left[u, A_{0}\right], \\
\partial_{t} v-\partial_{x} v & =\left[v, A_{0}\right], \\
\partial_{x} A_{0} & =\frac{1}{2}[v, u] .
\end{aligned}
$$

System (6)-(8) has the conservation of charge

$$
\begin{aligned}
& \int_{\mathbb{R}}|u(t, x)|^{2}+|v(t, x)|^{2} d x \\
& \quad=\int_{\mathbb{R}}|u(0, x)|^{2}+|v(0, x)|^{2} d x .
\end{aligned}
$$

From (8), we have the following representation, with a boundary condition $A_{0}(-\infty)=0$,

$$
A_{0}(t, x)=\frac{1}{2} \int_{-\infty}^{x}[v, u](t, y) d y .
$$

We have showed that the initial value problem of (6)-(8) reduces to the study of the following system

$$
\begin{gathered}
\partial_{t} u+\partial_{x} u=\left[u, A_{0}\right], \\
\partial_{t} v-\partial_{x} v=\left[v, A_{0}\right], \\
u(0, x)=u_{0}(x), \\
v(0, x)=v_{0}(x),
\end{gathered}
$$

where $A_{0}$ is defined by (10).

Theorem 1. For initial data $u_{0}, v_{0} \in H^{b}(\mathbb{R})(b=0,1)$, the initial value problem for (11) has a unique, global in time solution which belongs to

$$
u, v \in C\left([0, \infty) ; H^{b}(\mathbb{R})\right) .
$$

Moreover we get the following upper bound of $\mathrm{H}^{1}$ norm

$$
\|u(t)\|_{H^{1}}^{2}+\|v(t)\|_{H^{1}}^{2} \leq c \exp (c t) .
$$

We will derive some asymptotic behaviors of solutions to (11) with $u_{0}, v_{0} \in C_{c}(\mathbb{R})$. We refer to Remark 7 in Section 2.

Next we impose the temporal gauge condition $A_{0}=0$. Then we have

$$
\begin{aligned}
\partial_{t} \phi+\partial_{x} N & =\left[N, A_{1}\right], \\
\partial_{t} N+\partial_{x} \phi & =\left[\phi, A_{1}\right], \\
\partial_{t} A_{1} & =[N, \phi] .
\end{aligned}
$$

With the notations $\phi+N=u$ and $\phi-N=v$, system (14) can be rewritten as

$$
\begin{aligned}
\partial_{t} u+\partial_{x} u & =\left[u, A_{1}\right], \\
\partial_{t} v-\partial_{x} v & =\left[A_{1}, v\right], \\
\partial_{t} A_{1} & =\frac{1}{2}[u, v] .
\end{aligned}
$$

Theorem 2. For initial data $u_{0}, v_{0}, a_{0} \in H^{b}(\mathbb{R})(b=0,1)$, the initial value problem for (15)-(17) has a unique, global in time solution which belongs to

$$
u, v, A_{1} \in C\left([0, \infty) ; H^{b}(\mathbb{R})\right) .
$$

Moreover we get the following upper bound of $\mathrm{H}^{1}$ norm:

$$
\|u(t)\|_{H^{1}}^{2}+\|v(t)\|_{H^{1}}^{2}+\left\|A_{1}(t)\right\|_{H^{1}}^{2} \leq c \exp (c t) .
$$

We obtain the following result for the critical case $L^{1}$.

Theorem 3. For any $u_{0}, v_{0}, a_{0} \in L^{1}$, the initial value problem for (15)-(17) has a unique, global in time solution which belongs to

$$
u, v, A_{1} \in C\left([0, \infty) ; L^{1}\right) .
$$

Now we summarize the algebraic definition and properties used for the proof of Theorems 1-3. Let $\mathbf{g}$ be a Lie algebra of a matrix Lie group such as $\mathrm{SO}(n), \mathrm{SU}(n)$. We define the following norm for $A \in \mathbf{g}$ :

$$
|A|^{2}=\langle A, A\rangle=\operatorname{tr}\left(A^{*} A\right) .
$$

Let $\langle\cdot, \cdot\rangle$ and $[\cdot, \cdot]$ denote inner product induced by the trace norm and Lie bracket, respectively. Then we have, for matrices $A, B, C \in \mathbf{g}$,

$$
\begin{aligned}
\langle[A, B], A\rangle & =0, \\
\langle A,[B, C]\rangle & =\langle[A, B], C\rangle .
\end{aligned}
$$

Theorem 1 is proved in Section 2. We show Theorems 2 and 3 in Sections 3 and 4, respectively. We conclude this section by giving a few notations. We use the standard Sobolev space $W^{s, p}(\mathbb{R})$ with the norm $\|f\|_{W^{s, p(\mathbb{R})}}=\left\|\Lambda^{s} f\right\|_{L^{p}(\mathbb{R})}$, where $\left(\widehat{\Lambda^{s} f}\right)(\xi)=\left(1+|\xi|^{2}\right)^{s / 2} \widehat{f}(\xi)$ and $\widehat{f}$ denotes the Fourier transform of $f$. The space $H^{s}(\mathbb{R})$ denotes $W^{s, 2}(\mathbb{R})$. We define the space-time norm $\|f\|_{L_{T}^{p} L^{q}}=\left(\int_{0}^{T}\|f(t, \cdot)\|_{L^{q}(\mathbb{R})}^{p} d t\right)^{1 / p}$. We use $c$ to denote various constants. When we are interested in local solutions, we may assume that $T \leq 1$. Thus we shall replace smooth function of $T, C(T)$ by $C$. We use $A \lesssim B$ to denote an estimate of the form $A \leq c B$. 


\section{Proof of Theorem 1}

To show the existence of local solution to (6)-(8), we introduce a linear estimate (see [7]).

Lemma 4. Let $u_{ \pm}$be the solution to the inhomogeneous equation

$$
\begin{aligned}
\partial_{t} u_{ \pm} \pm \partial_{x} u_{ \pm} & =F_{ \pm}(t, x), \\
u_{ \pm}(0, x) & =u_{ \pm 0}(x),
\end{aligned}
$$

where $u_{ \pm 0} \in L^{p}$ and $F_{ \pm} \in L_{\text {loc }}^{1}\left([0, T), L^{p}(\mathbb{R})\right)$ for $1 \leq p \leq \infty$. Then for $T>0$ we have

$$
\left\|u_{ \pm}(t, \cdot)\right\|_{L^{p}} \leq\left\|u_{ \pm 0}\right\|_{L^{p}}+\int_{0}^{T}\left\|F_{ \pm}(s, \cdot)\right\|_{L^{p}} d s .
$$

We will prove the existence of local solution to (11) with $u_{0}, v_{0} \in L^{2}(\mathbb{R})$. The case of $u, v \in H^{1}(\mathbb{R})$ can be treated similarly. Proof follows by standard arguments from a priori estimates of the following Propositions.

Proposition 5. Let $u, v$ be the solution of (11) in a strip $[0, T] \times$ $\mathbb{R}$ with

$$
u, v \in C\left([0, T) ; L^{2}(\mathbb{R})\right)
$$

Define

$$
J(T)=\sup _{0 \leq t \leq T}\|u(t, \cdot)\|_{L^{2}(\mathbb{R})}+\|v(t, \cdot)\|_{L^{2}(\mathbb{R})} .
$$

Then there exist constants $c>0$ and $T^{*}>0$, depending only on $J(0)$, such that if $T<T^{*}$ then $J(T) \leq c J(0)$.

Proposition 6. Let $u, v$ and $u^{\prime}, v^{\prime}$ be two solutions of (11) verifying the hypothesis of Proposition 5 in a strip $\mathbb{R} \times[0, T]$ and let $J(T)$ as in a Proposition 5 and $J^{\prime}(T)$ be the corresponding quantity for the primed solution. Define

$$
\begin{aligned}
\Delta(T)= & \sup _{0 \leq t \leq T}\left\|u(t, \cdot)-u^{\prime}(t, \cdot)\right\|_{L^{2}(\mathbb{R})} \\
& +\left\|v(t, \cdot)-v^{\prime}(t, \cdot)\right\|_{L^{2}(\mathbb{R})} .
\end{aligned}
$$

Then there exist constants $c>0$ and $T^{*}>0$, depending only on $J(0)$ and $J^{\prime}(0)$, such that if $T<T^{*}$ then $\triangle(T) \leq c \triangle(0)$.

Proposition 6 follows by the similar argument to Proposition 5. We will only prove Proposition 5. We define

$$
\begin{aligned}
X(T)= & \int_{0}^{T}\left\|\left[u, A_{0}\right](s, \cdot)\right\|_{L^{2}(\mathbb{R})} \\
& +\left\|\left[v, A_{0}\right](s, \cdot)\right\|_{L^{2}(\mathbb{R})} d s .
\end{aligned}
$$

It is easily shown that $J(T) \lesssim J(0)+X(T)$ by applying Lemma 4 . We will derive the inequality $X(T) \lesssim T(J(0)+$ $X(T))^{3}$. Then the bootstrap argument completes the proof of Proposition 5. From representation (10), we have the following $L^{\infty}$ bound:

$$
\left\|A_{0}(t, \cdot)\right\|_{L^{\infty}(\mathbb{R})} \leq \frac{1}{2}\left(\|u(t, \cdot)\|_{L^{2}(\mathbb{R})}^{2}+\|v(t, \cdot)\|_{L^{2}(\mathbb{R})}^{2}\right) .
$$

Applying (29), the integrals in $X(T)$ can be treated as follows:

$$
\begin{aligned}
& \int_{0}^{T}\left\|\left[u, A_{0}\right](s, \cdot)\right\|_{L^{2}(\mathbb{R})} d s \lesssim T(J(0)+X(T))^{3}, \\
& \int_{0}^{T}\left\|\left[v, A_{0}\right](s, \cdot)\right\|_{L^{2}(\mathbb{R})} d s \lesssim T(J(0)+X(T))^{3} .
\end{aligned}
$$

Then we get the relation $X(T) \lesssim T(J(0)+X(T))^{3}$ which completes the proof of Proposition 5 by the bootstrap argument. Global existence for $L^{2}$ initial data can be proved taking charge conservation (9) into account.

We will prove the global existence of solutions to the initial problem (11) for initial data $u_{0}, v_{0} \in H^{1}(\mathbb{R})$. Differentiating both sides of (11), we can obtain

$$
\begin{aligned}
\left(\partial_{t}+\partial_{x}\right)\left|u_{x}\right|^{2} & =2\left\langle\left[u, \partial_{x} A_{0}\right], u_{x}\right\rangle \\
& \leq 4|u|\left|u_{x}\right|\left|\partial_{x} A_{0}\right|, \\
\left(\partial_{t}-\partial_{x}\right)\left|v_{x}\right|^{2} & =2\left\langle\left[v, \partial_{x} A_{0}\right], v_{x}\right\rangle \leq 4|v|\left|v_{x}\right|\left|\partial_{x} A_{0}\right| .
\end{aligned}
$$

Considering (10), we obtain

$$
\begin{aligned}
& \partial_{t}\left(\left|u_{x}\right|^{2}+\left|v_{x}\right|^{2}\right)+\partial_{x}\left(\left|u_{x}\right|^{2}-\left|v_{x}\right|^{2}\right) \\
& \quad \leq 4\left|\partial_{x} A_{0}\right|\left(|u|\left|u_{x}\right|+|v|\left|v_{x}\right|\right) \\
& \quad \leq 4|u v|\left(|u|\left|u_{x}\right|+|v|\left|v_{x}\right|\right) \\
& \quad \leq 2\left(|u|^{2}+|v|^{2}\right)\left(|u|\left|u_{x}\right|+|v|\left|v_{x}\right|\right) .
\end{aligned}
$$

Integrating on $\mathbb{R}$ and using Gagliardo-Nirenberg inequality

$$
\begin{aligned}
& \|u\|_{L^{6}(\mathbb{R})}^{3}+\|v\|_{L^{6}(\mathbb{R})}^{3} \\
& \quad \leq c\left(\|u\|_{L^{2}(\mathbb{R})}^{2}+\|v\|_{L^{2}(\mathbb{R})}^{2}\right)\left(\left\|u_{x}\right\|_{L^{2}(\mathbb{R})}+\left\|v_{x}\right\|_{L^{2}(\mathbb{R})}\right),
\end{aligned}
$$

we have

$$
\begin{aligned}
& \frac{d}{d t}\left(\left\|u_{x}\right\|_{L^{2}(\mathbb{R})}^{2}+\left\|v_{x}\right\|_{L^{2}(\mathbb{R})}^{2}\right) \\
& \quad \leq c\left(\|u\|_{L^{2}(\mathbb{R})}^{2}+\|v\|_{L^{2}(\mathbb{R})}^{2}\right)\left(\left\|u_{x}\right\|_{L^{2}(\mathbb{R})}^{2}+\left\|v_{x}\right\|_{L^{2}(\mathbb{R})}^{2}\right) .
\end{aligned}
$$

Then we obtain

$$
\begin{aligned}
& \left\|u_{x}\right\|_{L^{2}(\mathbb{R})}^{2}+\left\|v_{x}\right\|_{L^{2}(\mathbb{R})}^{2} \\
& \quad \leq \exp \left(c\left(\left\|u_{0}\right\|_{L^{2}(\mathbb{R})}^{2}+\left\|v_{0}\right\|_{L^{2}(\mathbb{R})}^{2}\right) t\right) \\
& \cdot\left(\left\|u_{x}(0, \cdot)\right\|_{L^{2}(\mathbb{R})}^{2}+\left\|v_{x}(0, \cdot)\right\|_{L^{2}(\mathbb{R})}^{2}\right) .
\end{aligned}
$$

Therefore we have an upper bound of $H^{1}$ norm to extend a local solution globally.

Remark 7. Let us consider the initial value problem of (6)(8) for initial data $u_{0}, v_{0}$ with compact $\operatorname{supp}\left(u_{0}\right), \operatorname{supp}\left(v_{0}\right) \in$ $[-R, R]$. Considering (22), we obtain from (11)

$$
\begin{aligned}
& \left(\partial_{t}+\partial_{x}\right)|u|^{2}=2\left\langle\left[u, A_{0}\right], u\right\rangle=0 \\
& \left(\partial_{t}-\partial_{x}\right)|v|^{2}=2\left\langle\left[v, A_{0}\right], v\right\rangle=0
\end{aligned}
$$


which implies

$$
\begin{gathered}
|u(t, x)|=\left|u_{0}(x-t)\right|, \\
|v(t, x)|=\left|v_{0}(x+t)\right| .
\end{gathered}
$$

Then we have

$$
[u, v](t, x)=0 \quad \text { for } t \geq R, x \in \mathbb{R},
$$

which implies $A_{0}(t, x)=0$ for $x \in \mathbb{R}, t \geq R$. Therefore we obtain the following system:

$$
\begin{aligned}
& \partial_{t} u+\partial_{x} u=0, \\
& \partial_{t} v-\partial_{x} v=0,
\end{aligned}
$$

with initial data $u(R, x)=a(x), v(R, x)=b(x)$. Hence we get the following solution to (39) for $t \geq R$ :

$$
\begin{aligned}
& u(t, x)=a(x-t), \\
& v(t, x)=b(x+t) .
\end{aligned}
$$

We can check a decay of the local $L^{2}$ norm in spite of conservation (9)

$$
\int_{l}^{r}\left(|u|^{2}+|v|^{2}\right)(t, x) d x \longrightarrow 0 \quad \text { as } t \longrightarrow \infty,
$$

where $[l, r]$ is any finite interval.

\section{Proof of Theorem 2}

We introduce the following bilinear estimates for the proof of existence of local solution to (15)-(17).

Lemma 8. Let $u_{ \pm}, A_{1}$ be the solution to inhomogeneous equations

$$
\begin{aligned}
\partial_{t} u_{ \pm} \pm \partial_{x} u_{ \pm} & =F_{ \pm}(t, x), \\
u_{ \pm}(0, x) & =u_{ \pm 0}(x), \\
\partial_{t} A_{1} & =G(t, x), \\
A_{1}(0, x) & =a_{1}(x),
\end{aligned}
$$

where $u_{ \pm 0}, a_{1} \in L^{p}$ and $F_{ \pm}(t, \cdot) \in L^{1}\left([0, T) ; L^{p}(\mathbb{R})\right), G(t, \cdot) \in$ $L^{1}\left([0, T) ; L^{p}(\mathbb{R})\right)$ for $1 \leq p \leq \infty$. Then the solutions $u_{ \pm}, A_{1}$ to (42) satisfy the following estimates:

$$
\begin{gathered}
\left\|u_{+} u_{-}\right\|_{L_{T^{p}}^{p} L^{p}} \leq\left(\frac{1}{2}\right)^{1 / p}\left(\left\|u_{+0}\right\|_{L^{p}}+\int_{0}^{T}\left\|F_{+}(s)\right\|_{L^{p}} d s\right) \\
\cdot\left(\left\|u_{-0}\right\|_{L^{p}}+\int_{0}^{T}\left\|F_{-}(s)\right\|_{L^{p}} d s\right), \\
\left\|u_{ \pm} A_{1}\right\|_{L_{T^{p}}^{p} L^{p}} \leq\left(\left\|u_{ \pm 0}\right\|_{L^{p}}+\int_{0}^{T}\left\|F_{ \pm}(s)\right\|_{L^{p}} d s\right) \\
\cdot\left(\left\|a_{1}\right\|_{L^{p}}+\int_{0}^{T}\|G(s)\|_{L^{p}} d s\right),
\end{gathered}
$$

for any $0<T<\infty$.
Proof. We refer to [7] for the proof of (43). We will show estimate (44). We have solution representations of $u_{ \pm}, A_{1}$

$$
\begin{aligned}
& u_{ \pm}(t, x)=u_{ \pm 0}(x \mp t)+\int_{0}^{t} F_{ \pm}(s, x \mp t \pm s) d s, \\
& A_{1}(t, x)=a_{1}(x)+\int_{0}^{t} G(s, x) d s .
\end{aligned}
$$

Applying change of variables and Fubini's theorem, we can derive the following four estimates:

$$
\begin{aligned}
& \left\|u_{ \pm 0}(x \mp t) a_{1}(x)\right\|_{L_{T}^{p} L^{p}}^{p} \\
& \leq \int_{\mathbb{R}^{2}}\left|u_{ \pm 0}(x \mp t) a_{1}(x)\right|^{p} d x d t \\
& =\int_{\mathbb{R}^{2}}\left|u_{ \pm 0}(y) a_{1}(z)\right|^{p} d y d z=\left\|u_{ \pm 0}\right\|_{L^{p}}^{p}\left\|a_{1}\right\|_{L^{p}}^{p}, \\
& \left\|u_{ \pm 0}(x \mp t) \int_{0}^{t} G(s, x) d s\right\|_{L_{T}^{p} L^{p}} \\
& \leq \int_{0}^{T}\left\|u_{ \pm 0}(x \mp t) G(s, x)\right\|_{L_{T^{p}}^{p}} d s \\
& \leq\left\|u_{ \pm 0}\right\|_{L^{p}} \int_{0}^{T}\|G(s)\|_{L^{p}} d s, \\
& \left\|a_{1}(x) \int_{0}^{t} F_{ \pm}(s, x \pm(s-t)) d s\right\|_{L_{T}^{p} L^{p}} \\
& \leq \int_{0}^{T}\left\|a_{1} F_{ \pm}(s, x \pm(s-t))\right\|_{L_{T}^{p} L^{p}} d s \\
& \leq\left\|a_{1}\right\|_{L^{p}} \int_{0}^{T}\left\|F_{ \pm}(s)\right\|_{L^{p}} d s, \\
& \left\|\int_{0}^{t} F_{ \pm}(s, x \pm(s-t)) d s \int_{0}^{t} G\left(s^{*}, x\right) d s^{*}\right\|_{L_{T}^{p} L^{p}} \\
& \leq \int_{0}^{T} \int_{0}^{T}\left\|F_{ \pm}(s, x \pm(s-t)) G\left(s^{*}, x\right)\right\|_{L_{T}^{p} L^{p}} d s d s^{*} \\
& \leq \int_{0}^{T}\left\|F_{ \pm}(s)\right\|_{L^{p}} d s \int_{0}^{T}\left\|G\left(s^{*}\right)\right\|_{L^{p}} d s^{*} .
\end{aligned}
$$

Combining the above estimates, we obtain the desired result (44).

We will prove the existence of local solution to (15)-(17) with $u_{0}, v_{0}, a_{1} \in L^{2}(\mathbb{R})$. Let us consider the following iteration system corresponding to (15)-(17):

$$
\begin{aligned}
\partial_{t} u^{(n+1)}+\partial_{x} u^{(n+1)} & =\left[u^{(n)}, A_{1}^{(n)}\right], \\
\partial_{t} v^{(n+1)}-\partial_{x} v^{(n+1)} & =\left[A_{1}^{(n)}, v^{(n)}\right], \\
\partial_{t} A_{1}^{(n+1)} & =\frac{1}{2}\left[u^{(n)}, v^{(n)}\right],
\end{aligned}
$$

with the initial data $u^{(n+1)}(0, x)=u_{0}(x), v^{(n+1)}(0, x)=v_{0}(x)$, $A_{1}^{(n+1)}(0, x)=a_{1}(x)$, and $u^{(0)}=v^{(0)}=A_{1}^{(0)}=0$. We will 
denote $R=2\left(\left\|u_{0}\right\|_{L^{2}}+\left\|v_{0}\right\|_{L^{2}}+\left\|a_{1}\right\|_{L^{2}}\right)$ and show the following boundedness of iteration:

$$
\begin{array}{r}
\left\|u^{(n)}\right\|_{L_{T}^{\infty} L^{2}} \leq R, \\
\left\|v^{(n)}\right\|_{L_{T}^{\infty} L^{2}} \leq R, \\
\left\|A_{1}^{(n)}\right\|_{L_{T}^{\infty} L^{2}} \leq R, \\
\left\|u^{(n)} A_{1}^{(n)}\right\|_{L_{T}^{2} L^{2}} \leq R^{2}, \\
\left\|A_{1}^{(n)} v^{(n)}\right\|_{L_{T}^{2} L^{2}} \leq R^{2}, \\
\left\|u^{(n)} v^{(n)}\right\|_{L_{T}^{2} L^{2}} \leq R^{2},
\end{array}
$$

for sufficiently small $T>0$. It is obvious for $n=0$.

Applying Lemma 4, it follows from Hölder inequality that

$$
\begin{aligned}
\left\|u^{(n+1)}\right\|_{L_{T}^{\infty} L^{2}} & \leq\left\|u_{0}\right\|_{L^{2}}+\int_{0}^{T}\left\|\left[u^{(n)}, A_{1}^{(n)}\right]\right\|_{L^{2}} d s \\
& \leq\left\|u_{0}\right\|_{L^{2}}+2 \sqrt{T}\left\|u^{(n)} A_{1}^{(n)}\right\|_{L_{T}^{2} L^{2}} \\
& \leq \frac{1}{2} R+2 \sqrt{T} R^{2} \leq R,
\end{aligned}
$$

for sufficiently small $T>0$. The estimate for $\left\|v^{(n+1)}\right\|_{L_{T}^{\infty} L^{2}} \leq R$ is similar. We also have from the third equation of (47)

$$
\begin{aligned}
\left\|A_{1}^{(n+1)}\right\|_{L_{T}^{\infty} L^{2}} & \leq\left\|a_{1}\right\|_{L^{2}}+\frac{1}{2} \int_{0}^{T}\left\|\left[u^{(n)}, v^{(n)}\right]\right\|_{L^{2}} d s \\
& \leq\left\|a_{1}\right\|_{L^{2}}+\sqrt{T}\left\|u^{(n)} v^{(n)}\right\|_{L_{T}^{2} L^{2}} \\
& \leq \frac{1}{2} R+\sqrt{T} R^{2} \leq R,
\end{aligned}
$$

for sufficiently small $T>0$. Then we obtain (48) for $n+1$ iteration.

We apply Lemma 8 to obtain (49)

$$
\begin{aligned}
& \left\|u^{(n+1)} A_{1}^{(n+1)}\right\|_{L_{T}^{2} L^{2}} \\
& \leq\left(\left\|u_{0}\right\|_{L^{2}}+2 \sqrt{T}\left\|u^{(n)} A_{1}^{(n)}\right\|_{L_{T}^{2} L^{2}} d s\right) \\
& \cdot\left(\left\|a_{1}\right\|_{L^{2}}+\sqrt{T}\left\|u^{(n)} v^{(n)}\right\|_{L_{T}^{2} L^{2}} d s\right) \leq R^{2},
\end{aligned}
$$

for sufficiently small $T>0$. Similarly one may obtain the estimate $\left\|A_{1}^{(n+1)} v^{(n+1)}\right\|_{L_{T^{2}} L^{2}}$ for small $T>0$. Applying Lemma 8 , we also have for sufficiently small $T>0$

$$
\begin{gathered}
\left\|u^{(n+1)} v^{(n+1)}\right\|_{L_{T}^{2} L^{2}} \\
\leq \frac{1}{\sqrt{2}}\left(\left\|u_{0}\right\|_{L^{2}}+2 \sqrt{T}\left\|u^{(n)} A_{1}^{(n)}\right\|_{L_{T}^{2} L^{2}} d s\right) \\
\cdot\left(\left\|v_{0}\right\|_{L^{2}}+2 \sqrt{T}\left\|A_{1}^{(n)} v^{(n)}\right\|_{L_{T}^{2} L^{2}} d s\right) \leq R^{2} .
\end{gathered}
$$

For the difference estimate we denote

$$
\begin{aligned}
d_{n}(T)= & \left\|u^{(n+1)}-u^{(n)}\right\|_{L_{T}^{\infty} L^{2}}+\left\|v^{(n+1)}-v^{(n)}\right\|_{L_{T}^{\infty} L^{2}} \\
& +\left\|A_{1}^{(n+1)}-A_{1}^{(n)}\right\|_{L_{T}^{\infty} L^{2}} \\
& +\left\|u^{(n+1)} A_{1}^{(n+1)}-u^{(n)} A_{1}^{(n)}\right\|_{L_{T}^{2} L^{2}} \\
& +\left\|A_{1}^{(n+1)} v^{(n+1)}-A_{1}^{(n)} v^{(n)}\right\|_{L_{T}^{2} L^{2}} \\
& +\left\|u^{(n+1)} v^{(n+1)}-u^{(n)} v^{(n)}\right\|_{L_{T}^{2} L^{2}} .
\end{aligned}
$$

We will show that for sufficiently small $T>0$

$$
d_{n}(T) \leq \frac{1}{2} d_{n-1}(T)
$$

which implies the existence of a local solution to (15)-(17).

Let us show how to estimate $\left\|u^{(n+1)}-u^{(n)}\right\|_{L_{T}^{\infty} L^{2}}, \| A_{1}^{(n+1)}-$ $A_{1}^{(n)}\left\|_{L_{T}^{\infty} L^{2}},\right\| u^{(n+1)} A_{1}^{(n+1)}-u^{(n)} A_{1}^{(n)} \|_{L_{T}^{2} L^{2}}$ and $\| u^{(n+1)} v^{(n+1)}-$ $u^{(n)} v^{(n)} \|_{L_{T}^{2} L^{2}}$. Other terms can be bounded in a similar way. Applying Lemma 4, we can derive from the first equation (48) that

$$
\begin{aligned}
& \left\|u^{(n+1)}-u^{(n)}\right\|_{L_{T}^{\infty} L^{2}} \\
& \quad \leq 2 \sqrt{T}\left\|u^{(n)} A_{1}^{(n)}-u^{(n-1)} A_{1}^{(n-1)}\right\|_{L_{T}^{2} L^{2}} .
\end{aligned}
$$

For $\left\|u^{(n+1)} A_{1}^{(n+1)}-u^{(n)} A_{1}^{(n)}\right\|_{L_{T}^{2} L^{2}}$, we estimate from Lemma 8

$$
\begin{aligned}
& \left\|u^{(n+1)} A_{1}^{(n+1)}-u^{(n)} A_{1}^{(n)}\right\|_{L_{T}^{2} L^{2}} \\
& \quad \leq\left\|u^{(n+1)}\left(A_{1}^{(n+1)}-A_{1}^{(n)}\right)\right\|_{L_{T}^{2} L^{2}} \\
& +\left\|\left(u^{(n+1)}-u^{(n)}\right) A_{1}^{(n)}\right\|_{L_{T}^{2} L^{2}} \\
& \quad \leq \sqrt{T}\left(\left\|u_{0}\right\|_{L^{2}}+2 \sqrt{T}\left\|u^{(n)} A_{1}^{(n)}\right\|_{L_{T}^{2} L^{2}}\right) \\
& \cdot\left\|u^{(n)} v^{(n)}-u^{(n-1)} v^{(n-1)}\right\|_{L_{T}^{2} L^{2}} \\
& +\sqrt{T}\left(\left\|a_{1}\right\|_{L^{2}}+\sqrt{T}\left\|^{(n)} v^{(n)}\right\|_{L_{T}^{2} L^{2}}\right) \\
& \quad\left\|u^{(n)} A_{1}^{(n)}-u^{(n-1)} A_{1}^{(n-1)}\right\|_{L_{T}^{2} L^{2}} \\
& \quad \leq \sqrt{T}\left(\frac{R}{2}+2 \sqrt{T} R^{2}\right) d_{n-1}(T) .
\end{aligned}
$$

We can derive

$$
\begin{aligned}
& \left\|A_{1}^{(n+1)}-A_{1}^{(n)}\right\|_{L_{T}^{\infty} L^{2}} \\
& \quad \leq \sqrt{T}\left\|u^{(n)} v^{(n)}-u^{(n-1)} v^{(n-1)}\right\|_{L_{T}^{2} L^{2}} .
\end{aligned}
$$


Applying Lemma 8, we have

$$
\begin{aligned}
& \left\|u^{(n+1)} v^{(n+1)}-u^{(n)} v^{(n)}\right\|_{L_{T}^{2} L^{2}} \\
& \quad \leq\left\|u^{(n+1)}\left(v^{(n+1)}-v^{(n)}\right)\right\|_{L_{T}^{2} L^{2}} \\
& +\left\|\left(u^{(n+1)}-u^{(n)}\right) v^{(n)}\right\|_{L_{T}^{2} L^{2}} \\
& \quad \leq \sqrt{\frac{T}{2}}\left(\left\|u_{0}\right\|_{L^{2}}+2 \sqrt{T}\left\|_{u^{(n)}} A_{1}^{(n)}\right\|_{L^{2}}\right) \\
& \cdot\left\|A_{1}^{(n)} v^{(n)}-A_{1}^{(n-1)} v^{(n-1)}\right\|_{L_{T}^{2} L^{2}} \\
& +\sqrt{\frac{T}{2}}\left(\left\|v_{0}\right\|_{L^{2}}+2 \sqrt{T}\left\|_{v^{(n)}} A_{1}^{(n)}\right\|_{L^{2}}\right) \\
& \cdot\left\|u^{(n)} A_{1}^{(n)}-u^{(n-1)} A_{1}^{(n-1)}\right\|_{L_{T}^{2} L^{2}} \\
& \quad \leq \sqrt{\frac{T}{2}}\left(\frac{R}{2}+2 \sqrt{T} R^{2}\right) d_{n-1}(T) .
\end{aligned}
$$

We can take care of other terms of $d_{n}(T)$ in the same manner as (56) and (57) to obtain

$$
d_{n}(T) \lesssim \sqrt{T}\left(1+\frac{R}{2}+2 \sqrt{T} R^{2}\right) d_{n-1}(T)
$$

We choose $T>0$ sufficiently small to obtain (55). Then we can prove the local existence, uniqueness, and Lipschitz continuity of solution by applying standard contraction mapping argument. Note that the existence time $T$ depends only on the size of norms of initial data.

Now we focus on global existence for $H^{1}(\mathbb{R})$ initial data. Differentiating both sides of (15)-(17), we can obtain

$$
\begin{aligned}
& \left(\partial_{t}+\partial_{x}\right)\left|u_{x}\right|^{2}=2\left\langle\left[u, \partial_{x} A_{1}\right], u_{x}\right\rangle \\
& \quad \leq 4|u|\left|u_{x}\right|\left|\partial_{x} A_{1}\right| \\
& \left(\partial_{t}-\partial_{x}\right)\left|v_{x}\right|^{2}=2\left\langle\left[\partial_{x} A_{1}, v\right], v_{x}\right\rangle \leq 4|v|\left|v_{x}\right|\left|\partial_{x} A_{1}\right| \\
& \partial_{t}\left|\partial_{x} A_{1}\right|^{2} \\
& \quad=\frac{1}{2}\left(\left\langle\partial_{x}[u, v], \partial_{x} A_{1}\right\rangle+\left\langle\partial_{x} A_{1}, \partial_{x}[u, v]\right\rangle\right) \\
& \leq 2\left(\left|u_{x}\right||v|\left|\partial_{x} A_{1}\right|+|u|\left|v_{x}\right|\left|\partial_{x} A_{1}\right|\right) .
\end{aligned}
$$

Integrating (61) on $\mathbb{R}$, we obtain

$$
\begin{gathered}
\frac{d}{d t}\left(\left\|u_{x}\right\|_{L^{2}(\mathbb{R})}^{2}+\left\|v_{x}\right\|_{L^{2}(\mathbb{R})}^{2}\right) \leq 2\left(\|u\|_{L^{\infty}(\mathbb{R})}+\|v\|_{L^{\infty}(\mathbb{R})}\right) \\
\cdot\left(\left\|\partial_{x} A_{1}\right\|_{L^{2}(\mathbb{R})}^{2}+\left\|u_{x}\right\|_{L^{2}(\mathbb{R})}^{2}+\left\|v_{x}\right\|_{L^{2}(\mathbb{R})}^{2}\right) .
\end{gathered}
$$

Integrating (62) on $\mathbb{R}$, we obtain

$$
\begin{gathered}
\frac{d}{d t}\left\|\partial_{x} A_{1}\right\|_{L^{2}(\mathbb{R})}^{2} \leq\left(\|u\|_{L^{\infty}(\mathbb{R})}+\|v\|_{L^{\infty}(\mathbb{R})}\right) \\
\cdot\left(\left\|u_{x}\right\|_{L^{2}(\mathbb{R})}^{2}+\left\|v_{x}\right\|_{L^{2}(\mathbb{R})}^{2}+\left\|\partial_{x} A_{1}\right\|_{L^{2}(\mathbb{R})}^{2}\right) .
\end{gathered}
$$

Combining (63) and (64), we arrive at

$$
\begin{aligned}
& \frac{d}{d t}\left(\left\|u_{x}\right\|_{L^{2}(\mathbb{R})}^{2}+\left\|v_{x}\right\|_{L^{2}(\mathbb{R})}^{2}+\left\|\partial_{x} A_{1}\right\|_{L^{2}(\mathbb{R})}^{2}\right) \\
& \quad \leq 3\left(\|u\|_{L^{\infty}(\mathbb{R})}+\|v\|_{L^{\infty}(\mathbb{R})}\right) \\
& \quad \cdot\left(\left\|u_{x}\right\|_{L^{2}(\mathbb{R})}^{2}+\left\|v_{x}\right\|_{L^{2}(\mathbb{R})}^{2}+\left\|\partial_{x} A_{1}\right\|_{L^{2}(\mathbb{R})}^{2}\right) .
\end{aligned}
$$

We estimate $L^{\infty}$ bound from (15), (16)

$$
\begin{aligned}
& \|u(t)\|_{L^{\infty}} \leq\left\|u_{0}\right\|_{L^{\infty}} \lesssim\left\|u_{0}\right\|_{H^{1}}, \\
& \|v(t)\|_{L^{\infty}} \leq\left\|v_{0}\right\|_{L^{\infty}} \lesssim\left\|v_{0}\right\|_{H^{1}} .
\end{aligned}
$$

Then Gronwall's inequality leads us to

$$
\left\|u_{x}\right\|_{L^{2}(\mathbb{R})}^{2}+\left\|v_{x}\right\|_{L^{2}(\mathbb{R})}^{2}+\left\|\partial_{x} A_{1}\right\|_{L^{2}(\mathbb{R})}^{2} \leq c e^{c t} .
$$

\section{Proof of Theorem 3}

We consider the critical space $L^{1}$. We will show the local existence of solution for small data and construct the local solution for the initial data of arbitrary size applying the finite speed of propagation of solution. Applying the nonconcentration property in the Lebesgue space which was introduced originally in [8], we will extend the local solution globally.

First we prove the existence of local solution for a small data. We will show the following boundedness by induction with respect to $n$.

$$
\begin{array}{r}
\left\|u^{(n)}\right\|_{L_{T}^{\infty} L^{1}} \leq R, \\
\left\|v^{(n)}\right\|_{L_{T}^{\infty} L^{1}} \leq R, \\
\left\|A_{1}^{(n)}\right\|_{L_{T}^{\infty} L^{1}} \leq R \\
\left\|u^{(n)} A_{1}^{(n)}\right\|_{L_{T}^{1} L^{1}} \leq R^{2}, \\
\left\|A_{1}^{(n)} v^{(n)}\right\|_{L_{T}^{1} L^{1}} \leq R^{2}, \\
\left\|u^{(n)} v^{(n)}\right\|_{L_{T}^{1} L^{1}} \leq R^{2},
\end{array}
$$

for sufficiently small $R>0$. It is obvious for $n=0$.

Applying Lemma 4, we have

$$
\begin{aligned}
\left\|u^{(n+1)}\right\|_{L_{T}^{\infty} L^{1}} & \leq\left\|u_{0}\right\|_{L^{1}}+2\left\|u^{(n)} A_{1}^{(n)}\right\|_{L_{T}^{1} L^{1}} \leq \frac{R}{2}+2 R^{2} \\
& \leq R
\end{aligned}
$$

for sufficiently small $R>0$. The estimate for $\left\|v^{(n+1)}\right\|_{L_{T}^{\infty} L^{1}}$ is similar. We also have

$$
\left\|A_{1}^{(n+1)}\right\|_{L_{T}^{\infty} L^{1}} \leq\left\|a_{1}\right\|_{L^{1}}+\left\|u^{(n)} v^{(n)}\right\|_{L_{T}^{1} L^{1}} \leq \frac{R}{2}+R^{2} \leq R,
$$

for sufficiently small $R>0$. 
Applying Lemma 8, we have

$$
\begin{gathered}
\left\|u^{(n+1)} A_{1}^{(n+1)}\right\|_{L_{T}^{1} L^{1}} \leq\left(\left\|u_{0}\right\|_{L^{1}}+2\left\|u^{(n)} A_{1}^{(n)}\right\|_{L^{1}} d s\right) \\
\cdot\left(\left\|a_{1}\right\|_{L^{1}}+\left\|u^{(n)} v^{(n)}\right\|_{L^{1}} d s\right) \leq R^{2},
\end{gathered}
$$

for sufficiently small $R>0$. The estimate for $\left\|A_{1}^{(n+1)} v^{(n+1)}\right\|_{L_{T}^{1} L^{1}}$ is similar. Applying Lemma 8, we also have, for sufficiently small $R>0$,

$$
\begin{gathered}
\left\|u^{(n+1)} v^{(n+1)}\right\|_{L_{T}^{1} L^{1}} \leq \frac{1}{2}\left(\left\|u_{0}\right\|_{L^{1}}+2\left\|u^{(n)} A_{1}^{(n)}\right\|_{L^{1}} d s\right) \\
\cdot\left(\left\|v_{0}\right\|_{L^{1}}+\left\|A_{1}^{(n)} v^{(n)}\right\|_{L^{1}} d s\right) \leq R^{2} .
\end{gathered}
$$

For the difference estimate we denote

$$
\begin{aligned}
d_{n}(T)= & \left\|u^{(n+1)}-u^{(n)}\right\|_{L_{T}^{\infty} L^{1}}+\left\|v^{(n+1)}-v^{(n)}\right\|_{L_{T}^{\infty} L^{1}} \\
& +\left\|A_{1}^{(n+1)}-A_{1}^{(n)}\right\|_{L_{T}^{\infty} L^{1}} \\
& +4\left\|u^{(n+1)} A_{1}^{(n+1)}-u^{(n)} A_{1}^{(n)}\right\|_{L_{T}^{1} L^{1}} \\
& +4\left\|A_{1}^{(n+1)} v^{(n+1)}-A_{1}^{(n)} v^{(n)}\right\|_{L_{T}^{1} L^{1}} \\
& +2\left\|u^{(n+1)} v^{(n+1)}-u^{(n)} v^{(n)}\right\|_{L_{T}^{1} L^{1}} .
\end{aligned}
$$

We will show that for sufficiently small $R>0$

$$
d_{n}(T) \leq \frac{1}{2} d_{n-1}(T),
$$

which implies the existence of a local solution to (15)-(17).

Let us show how to estimate $\left\|u^{(n+1)}-u^{(n)}\right\|_{L_{T}^{\infty} L^{1}}, \| A_{1}^{(n+1)}$ $A_{1}^{(n)}\left\|_{L_{T}^{\infty} L^{1}},\right\| u^{(n+1)} A_{1}^{(n+1)}-u^{(n)} A_{1}^{(n)} \|_{L_{T}^{1} L^{1}}$, and $\| u^{(n+1)} v^{(n+1)}-$ $u^{(n)} v^{(n)} \|_{L_{T}^{1} L^{1}}$. Other terms can be bounded in a similar way. Applying Lemma 4, we can derive

$$
\left\|u^{(n+1)}-u^{(n)}\right\|_{L_{T}^{\infty} L^{1}} \leq 2\left\|u^{(n)} A_{1}^{(n)}-u^{(n-1)} A_{1}^{(n-1)}\right\|_{L_{T}^{1} L^{1}} .
$$

For $\left\|u^{(n+1)} A_{1}^{(n+1)}-u^{(n)} A_{1}^{(n)}\right\|_{L_{T}^{1} L^{1}}$, we estimate from Lemma 8

$$
\begin{aligned}
& \left\|u^{(n+1)} A_{1}^{(n+1)}-u^{(n)} A_{1}^{(n)}\right\|_{L_{T}^{1} L^{1}} \\
& \leq\left\|u^{(n+1)}\left(A_{1}^{(n+1)}-A_{1}^{(n)}\right)\right\|_{L_{T}^{1} L^{1}} \\
& +\left\|\left(u^{(n+1)}-u^{(n)}\right) A_{1}^{(n)}\right\|_{L_{T}^{1} L^{1}} \\
& \leq\left(\left\|u_{0}\right\|_{L^{1}}+2\left\|u^{(n)} A_{1}^{(n)}\right\|_{L^{1}}\right) \\
& \cdot\left\|u^{(n)} v^{(n)}-u^{(n-1)} v^{(n-1)}\right\|_{L_{T}^{1} L^{1}} \\
& +\left(\left\|a_{1}\right\|_{L^{1}}+\left\|u^{(n)} v^{(n)}\right\|_{L^{1}}\right) \\
& \cdot\left\|u^{(n)} A_{1}^{(n)}-u^{(n-1)} A_{1}^{(n-1)}\right\|_{L_{T}^{1} L^{1}} \\
& \quad \leq \frac{1}{2}\left(\frac{R}{2}+2 R^{2}\right) d_{n-1}(T) .
\end{aligned}
$$

We can derive

$$
\left\|A_{1}^{(n+1)}-A_{1}^{(n)}\right\|_{L_{T}^{\infty} L^{1}} \leq\left\|u^{(n)} v^{(n)}-u^{(n-1)} v^{(n-1)}\right\|_{L_{T}^{1} L^{1}} .
$$

For $\left\|u^{(n+1)} v^{(n+1)}-u^{(n)} v^{(n)}\right\|_{L_{T}^{1} L^{1}}$, we estimate from Lemma 8

$$
\begin{aligned}
& \left\|u^{(n+1)} v^{(n+1)}-u^{(n)} v^{(n)}\right\|_{L_{T}^{1} L^{1}} \\
& \leq\left\|u^{(n+1)}\left(v^{(n+1)}-v^{(n)}\right)\right\|_{L_{T}^{1} L^{1}} \\
& +\left\|\left(u^{(n+1)}-u^{(n)}\right) v^{(n)}\right\|_{L_{T}^{1} L^{1}} \\
& \leq \frac{1}{2}\left(\left\|u_{0}\right\|_{L^{1}}+2\left\|u^{(n)} A_{1}^{(n)}\right\|_{L^{1}}\right) \\
& \cdot\left\|A_{1}^{(n)} v^{(n)}-A_{1}^{(n-1)} v^{(n-1)}\right\|_{L_{T}^{2} L^{2}} \\
& +\frac{1}{2}\left(\left\|v_{0}\right\|_{L^{1}}+2\left\|v^{(n)} A_{1}^{(n)}\right\|_{L^{1}}\right) \\
& \cdot\left\|u^{(n)} A_{1}^{(n)}-u^{(n-1)} A_{1}^{(n-1)}\right\|_{L_{T}^{1} L^{1}} \\
& \quad \leq \frac{1}{8}\left(\frac{R}{2}+2 R^{2}\right) d_{n-1}(T) .
\end{aligned}
$$

We can take care of other terms of $d_{n}(T)$ in the same manner as (75)-(78) to obtain

$$
d_{n}(T) \leq \frac{1}{2}\left(1+\frac{R}{2}+2 R^{2}\right) d_{n-1}(T) .
$$

Next we make use of the finite speed of propagation of solution to construct the local solution for the initial data of arbitrary size. Choose $r$ satisfying

$$
\sup _{x \in \mathbb{R}} \int_{|x-y|<r}\left|u_{0}(y)\right|+\left|v_{0}(y)\right|+\left|a_{1}(y)\right| d y<R .
$$

With this $r$, let us split $\mathbb{R}$ in two ways

$$
\begin{aligned}
& I_{1}^{j}=[(2 j-1) r,(2 j+1) r], \\
& I_{2}^{j}=[2 j r, 2(j+1) r],
\end{aligned}
$$

for each $j \in \mathbb{Z}$. We also denote that

$$
\begin{aligned}
& \Omega_{1}^{j}:=\left\{(t, x) \in \mathbb{R}^{2}:|x-2 j r| \leq r-t, t \geq 0\right\}, \\
& \Omega_{2}^{j}:=\left\{(t, x) \in \mathbb{R}^{2}:|x-(2 j+1) r| \leq r-t, t \geq 0\right\} .
\end{aligned}
$$

Applying the finite speed of propagation and considering the smallness condition (80), we can construct the corresponding solution $u^{j, k}, v^{j, k}, A_{1}^{j, k}$ in $\Omega_{k}^{j}$, for each $I_{k}^{j}$. Let us consider the following regions:

$$
\begin{aligned}
& S_{1}^{j}=\left[0, \frac{r}{2}\right) \times\left[\left(2 j-\frac{1}{2}\right) r,\left(2 j+\frac{1}{2}\right) r\right], \\
& S_{2}^{j}=\left[0, \frac{r}{2}\right) \times\left[\left(2 j+\frac{1}{2}\right) r,\left(2 j+\frac{3}{2}\right) r\right] .
\end{aligned}
$$


Then we get a solution $u^{j, k}, v^{j, k}, A_{1}^{j, k}$ on $S_{k}^{j}$. Note that this solution is not influenced by changing the initial data on the complement of $I_{k}^{j}$. Using the uniqueness of solution we can gather these solutions to obtain a solution $u, v, A_{1}$ on $\bigcup_{k=1,2} \bigcup_{j \in Z} \Omega_{k}^{j}$. Then we know that the solution $u, v, A_{1}$ belongs to $L_{T}^{1} L^{1}(\mathbb{R})$.

To complete Theorem 3, we will show the nonconcentration property of the solution to extend the local solution globally. Let $T$ be the maximal time of the existence such that the solution $u, v, A_{1} \in L_{T}^{1} L^{1}(\mathbb{R})$ to (15)-(17) with initial data $u_{0}, v_{0}, a_{1}$. Now we assume that $T<\infty$. We will show that there exists $r=r\left(T,\left\|u_{0}\right\|_{L^{1}},\left\|v_{0}\right\|_{L^{1}},\left\|a_{1}^{0}\right\|_{L^{1}}\right)>0$ such that

$$
\begin{aligned}
& \sup _{0<t<T} \sup _{x \in \mathbb{R}} \int_{|x-y|<r}|u(t, y)|+|v(t, y)|+\left|A_{1}(t, y)\right| d y \\
& \quad<\varepsilon
\end{aligned}
$$

for any $\varepsilon>0$. If condition (84) holds, then there exist $0<$ $t^{*}<T$ and $r^{*}=r^{*}\left(T,\left\|u\left(t^{*}\right)\right\|_{L^{1}},\left\|v\left(t^{*}\right)\right\|_{L^{1}},\left\|A_{1}\left(t^{*}\right)\right\|_{L^{1}}\right)>0$ satisfying

$$
\begin{aligned}
& \sup _{x \in \mathbb{R}} \int_{|x-y|<r^{*}}\left|u\left(t^{*}, y\right)\right|+\left|v\left(t^{*}, y\right)\right|+\left|A_{1}\left(t^{*}, y\right)\right| d y \\
& \quad<R .
\end{aligned}
$$

Taking a new initial data $\left(u\left(t^{*}\right), v\left(t^{*}\right), A_{1}\left(t^{*}\right)\right)$, we can extend the solution over $[0, T]$ which gives a contradiction. Therefore we must have $T=\infty$ and then prove the existence of global solution.

Let $\left(u, v, A_{1}\right)$ be the smooth solution to (15)-(17). From (15) and (16), we obtain

$$
\begin{aligned}
& \left(\partial_{t}+\partial_{x}\right)|u|^{2}=2\left\langle\left[u, A_{1}\right], u\right\rangle=0, \\
& \left(\partial_{t}-\partial_{x}\right)|v|^{2}=2\left\langle\left[A_{1}, v\right], v\right\rangle=0,
\end{aligned}
$$

where (22) is taken into account. Then we derive

$$
\begin{gathered}
|u(t, x)|=\left|u_{0}(x-t)\right|, \\
|v(t, x)|=\left|v_{0}(x+t)\right|,
\end{gathered}
$$

from which we have

$$
\begin{aligned}
& \sup _{x \in \mathbb{R}} \int_{|x-y|<r}|u(t, y)| d y \leq \sup _{x \in \mathbb{R}} \int_{|x-y|<r}\left|u_{0}(y-t)\right| d y, \\
& \sup _{x \in \mathbb{R}} \int_{|x-y|<r}|v(t, y)| d y \leq \sup _{x \in \mathbb{R}} \int_{|x-y|<r}\left|v_{0}(y+t)\right| d y .
\end{aligned}
$$

From (17), we derive

$$
\begin{aligned}
\left|A_{1}(t, x)\right| & \leq\left|a_{1}(x)\right|+\frac{1}{2} \int_{0}^{t}|[u, v](s, x)| d s \\
& \leq\left|a_{1}(x)\right|+\int_{0}^{t}\left|u_{0}(x-s)\right|\left|v_{0}(x+s)\right| d s,
\end{aligned}
$$

where (87) is used. Integrating on $|x-y|<r$ and applying change of variables $y-s=z, y+s=w$ and Fubini's theorem, we have

$$
\begin{aligned}
& \int_{x-r}^{x+r} \int_{0}^{t}\left|u_{0}(y-s)\right|\left|v_{0}(y+s)\right| d s d y \\
& \quad \leq \int_{x-r-t}^{x+r} \int_{2 x-z-2 r}^{2 x-z+2 r}\left|u_{0}(z)\right|\left|v_{0}(\omega)\right| d \omega d z \\
& \quad \leq \int_{x-r-t}^{x+r}\left|u_{0}(z)\right|\left(\int_{2 x-z-2 r}^{2 x-z+2 r}\left|v_{0}(\omega)\right| d \omega\right) d z \\
& \left.\quad \leq \sup _{y \in \mathbb{R}} \int_{|y-\omega|<2 r}\left|v_{0}(\omega)\right| d \omega\right)\left(\int_{\mathbb{R}}\left|u_{0}(z)\right| d z\right) .
\end{aligned}
$$

Then we obtain

$$
\begin{aligned}
& \sup _{x \in \mathbb{R}} \int_{|x-y|<r}\left|A_{1}(t, y)\right| d y \\
& \leq \sup _{x \in \mathbb{R}} \int_{|x-y|<r}\left|a_{1}(y)\right| d y \\
& \quad+\sup _{x \in \mathbb{R}} \int_{|x-y|<r} \int_{0}^{t}\left|u_{0}(y-s)\right|\left|v_{0}(y+s)\right| d s d y \\
& \leq \sup _{x \in \mathbb{R}} \int_{|x-y|<r}\left|a_{1}(y)\right| d y \\
& \quad+\left\|u_{0}\right\|_{L^{1}}\left(\sup _{y \in \mathbb{R}} \int_{|x-y|<2 r}\left|v_{0}(\omega)\right| d \omega\right) .
\end{aligned}
$$

We can verify the nonconcentration of solution (84) for taking $r$ sufficiently small in (88) and (91).

\section{Competing Interests}

The authors declare that there is no conflict of interests regarding the publication of this article and regarding the funding that they have received.

\section{Acknowledgments}

Hyungjin Huh was supported by Basic Science Research Program through the National Research Foundation of Korea (NRF) funded by the Ministry of Education (2014R1A1A2053747).

\section{References}

[1] B. Dai, C.-L. Terng, and K. Uhlenbeck, "On the space-time monopole equation," Surveys in Differential Geometry, vol. 10, pp. 1-30, 2006.

[2] R. S. Ward, “Twistors in $2+1$ dimensions," Journal of Mathematical Physics, vol. 30, no. 10, pp. 2246-2251, 1989.

[3] M. Czubak, "Local wellposedness for the 2+1-dimensional monopole equation," Analysis \& PDE, vol. 3, no. 2, pp. 151-174, 2010.

[4] N. Bournaveas and T. Candy, "Local well-posedness for the space-time monopole equation in Lorenz gauge," NoDEA. 
Nonlinear Differential Equations and Applications, vol. 19, no. 1, pp. 67-78, 2012.

[5] A. Tesfahun, "Almost critical local well-posedness for the spacetime monopole equation in Lorenz gauge," Communications in Contemporary Mathematics, vol. 17, no. 3, Article ID 1450043, 14 pages, 2015.

[6] H. Huh and J. Yim, "Global solutions to space-time monopole equations in one space dimension," Journal of Mathematical Analysis and Applications, vol. 432, no. 1, pp. 74-85, 2015.

[7] S. Machihara and T. Ogawa, "Global well-posedness for one dimensional Chern-Simons-Dirac system in Lp," https://arxiv .org/abs/1601.07722.

[8] T. Candy, "Global existence for an $L^{2}$ critical nonlinear Dirac equation in one dimensio," Advances in Differential Equations, vol. 16, no. 7-8, pp. 643-666, 2011. 


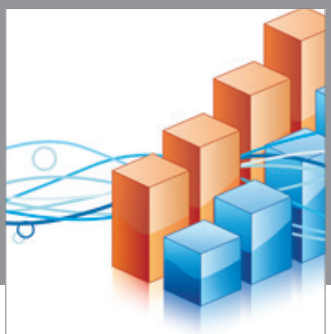

Advances in

Operations Research

vatem alat4

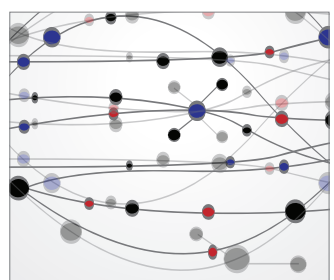

\section{The Scientific} World Journal
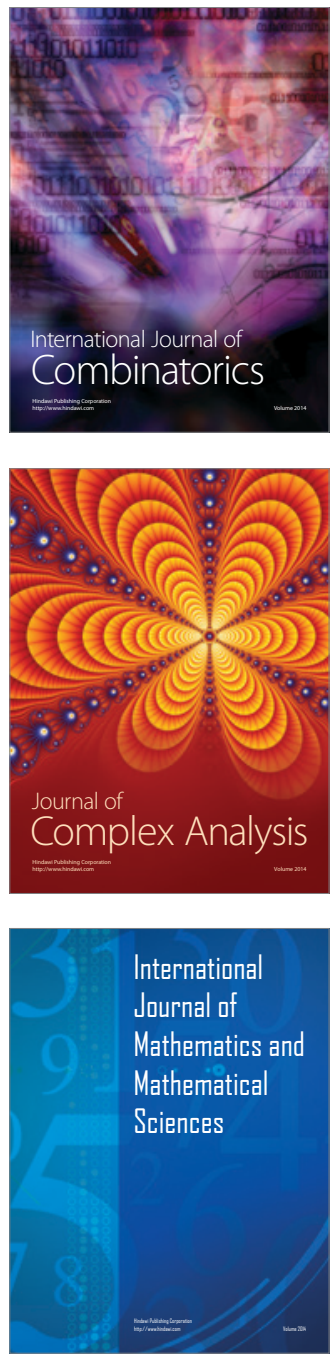
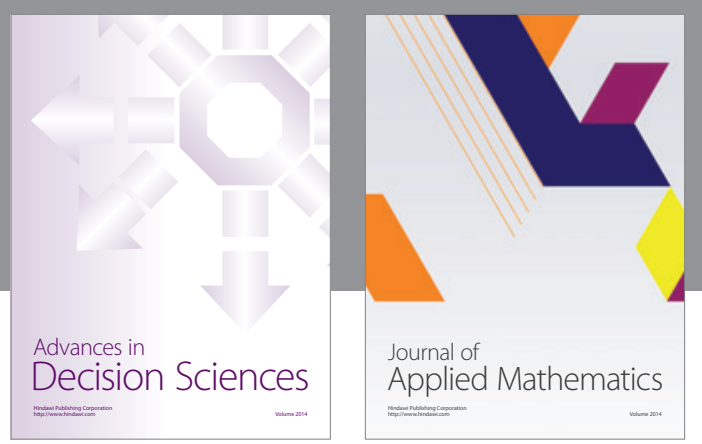

Algebra

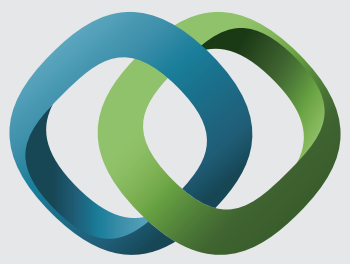

\section{Hindawi}

Submit your manuscripts at

https://www.hindawi.com
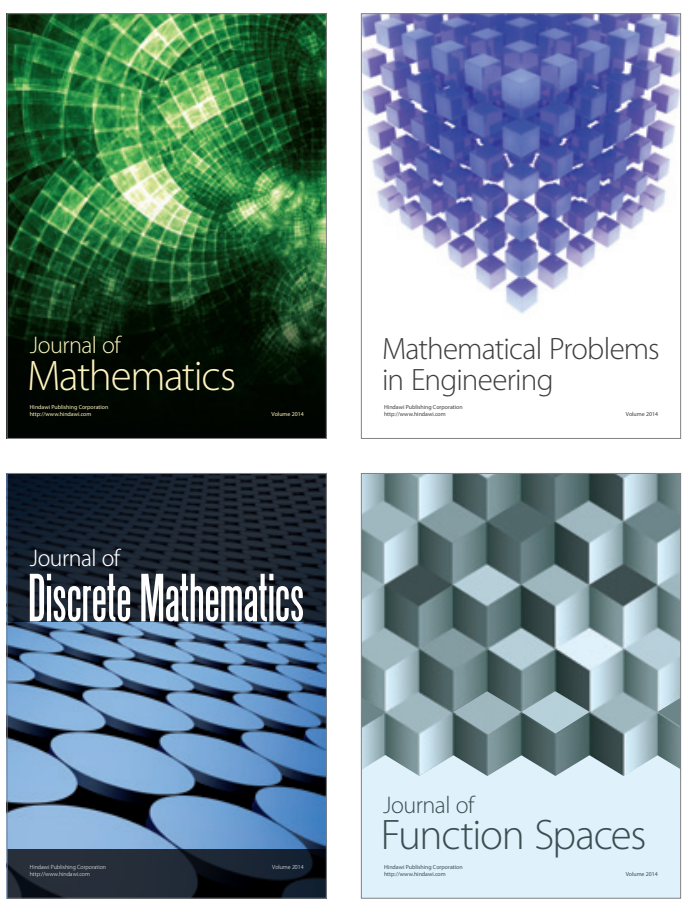

Mathematical Problems in Engineering
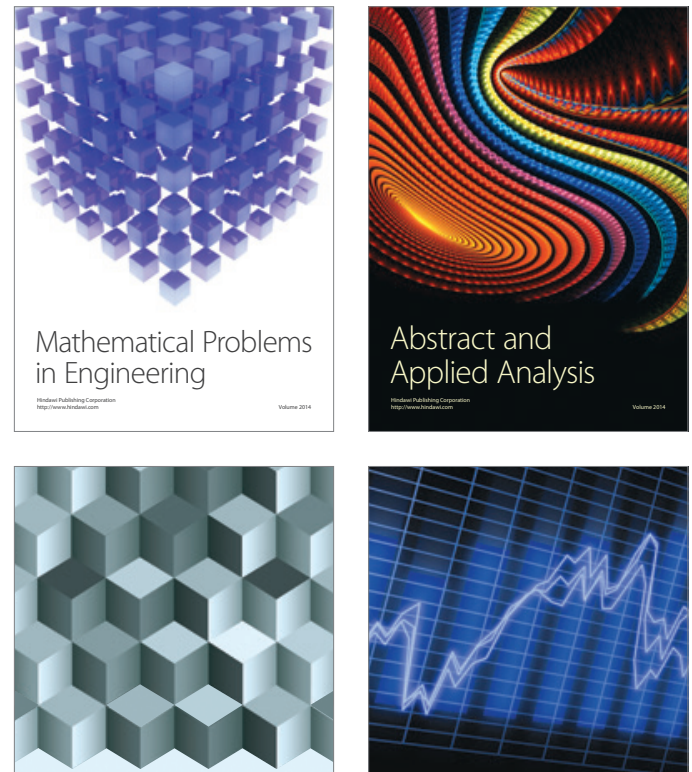

Journal of

Function Spaces

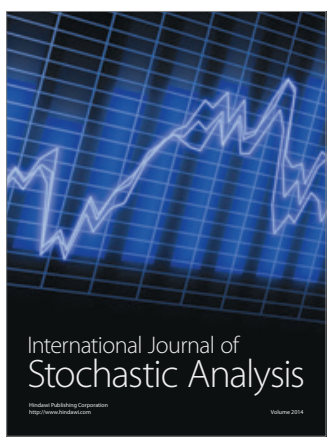

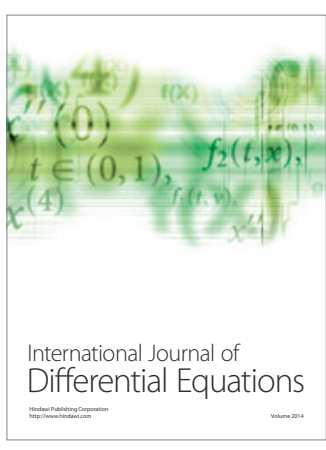
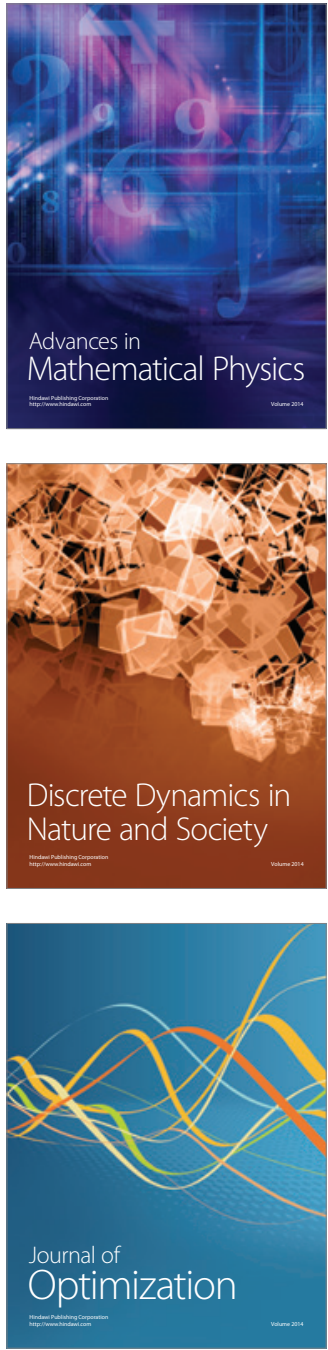